\title{
HIGHLIGHTS
}

PENILE CANCER

\section{New nomogram accurately predicts risk of metastasis in men with clinically negative lymph nodes}

Researchers at the Fudan University Shanghai Cancer Center in China have designed and tested a nomogram that outperforms the European Association of Urology (EAU) risk classification in identifying patients with penile cancer at high risk of occult lymph node metastasis. "We wanted to provide a risk-based approach for better selection of treatment options," says lead author Yao Zhu.

Early detection and treatment of metastatic lymph nodes in men with penile carcinoma is imperative given the poor survival associated with untreated disease. However, it has also been estimated that $80 \%$ of patients undergo unnecessary intervention, such as prophylactic lymphadenectomy and dynamic sentinel lymph node biopsy.

Clearly, better patient selection is required.

Zhu and colleagues gathered

clinicopathological information from 110 men with penile squamous cell carcinoma and clinically negative inguinal lymph nodes (defined as either impalpable nodes or palpable nodes that were negative on fine needle aspiration biopsy). T stage, grade, lymphovascular invasion and p53 expression were found to be associated with positive lymph node status on pathology, and so these features were used to construct a nomogram.

Bootstrap and decision curve analyses demonstrated the nomogram's superiority over EAU guidelines for selecting patients for early lymphadenectomy. The c-value of the nomogram was 0.79 , compared to 0.71 for the EAU model, indicating good correlation between predicted risk and observed incidence. Using a threshold probability of $10 \%$ to represent the risk of metastasis at which a patient would choose intervention, the nomogram correctly identified the proportion of patients at high risk and decreased interventions by 13 cases per 100 .

Zhu and his team plan to perform prospective evaluation studies within their

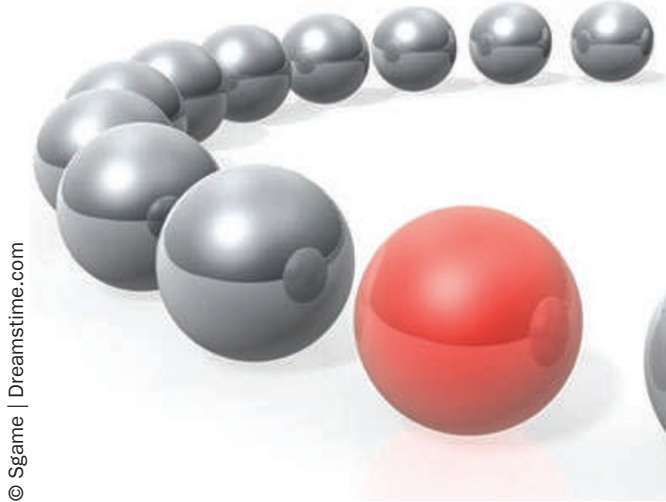

clinical practice to further improve the prognostic accuracy of the nomogram, before performing a multicenter study for external validation.

Sarah Payton

\footnotetext{
Original article Zhu, Y. et al. Development and evaluation of a nomogram to predict inguinal lymph node metastasis in patients with penile cancer and clinically negative lymph nodes. J. Urol. 184, 539-545 (2010)
} 